Dom. Cien., ISSN: 2477-8818

Vol. 4, núm. 4, octubre, 2018, pp. 145-158

El marketing sensorial y su influencia en el comportamiento de compra de los consumidores de la ciudad de Manta,

LAMIN CIENCIAS Ecuador

Número Publicado el 5 de octubre de 2018

DOI: $10.23857 /$ dc.v4i4.828

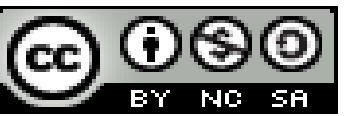

Ciencias económicas y empresariales

Artículo de investigación

\title{
El marketing sensorial y su influencia en el comportamiento de compra de los consumidores de la ciudad de Manta, Ecuador
}

\section{Sensory marketing and its influence on the buying behavior of consumers in the city of Manta, Ecuador}

\section{Marketing sensorial e sua influência no comportamento de compra de consumidores na cidade de Manta, Equador}

\begin{abstract}
Xiomara M. Palma-Perez ${ }^{\mathrm{I}}$
madelaine.palma@hotmail.com

Rossanna K. Arteaga-Flores II

rossannaartega.artega@uleam.edu.ec

Johnny E. Ponce-Andrade III

johnny.ponce@uleam.edu.ec
\end{abstract}

\begin{abstract}
Tomistocles D. Loor- Chávez IV temístocles.loor@uleam.edu.ec José C. Calderón Bailón $\mathrm{V}$ jose.calderon@uleam.edu.ec Pericles R. Mejía Vásquez VI pericles.mejia@uleam.edu.ec
\end{abstract}

Recibido: 14 de julio de 2018 * Corregido: 05 de agosto de $2018 *$ Aceptado: 17 de septiembre de 2018

\footnotetext{
Ingeniera en Marketing, graduada de la Universidad Laica Eloy Alfaro de Manabí, Manta, Ecuador.

II. Magíster en Negocios Internacionales y Gestión en Comercio Exterior, Magíster en Investigación en Administración y Economía de la Empresa, Economista, Docente Universidad Eloy Alfaro de Manabí, Manta, Ecuador.

III. Doctor en Ciencias Administrativas, Magister en Dirección Estratégica de Marketing y Ventas, Especialista en Gerencia Estratégica de la Comunicación Organizacional, Ingeniero Comercial, Docente Universidad Eloy Alfaro de Manabí, Manta, Ecuador.

IV. Doctor en Ciencias Administrativas. Magister en Dirección Universitaria, Ingeniero Comercial, Docente Universidad Eloy Alfaro de Manabí, Manta, Ecuador.

v. Magíster en Administración de Empresas con Mención en Marketing, Licenciado en Comunicación Especialidad Publicidad, Docente Universidad Eloy Alfaro de Manabí, Manta, Ecuador. Doctorando Universidad de Córdoba deEspaña.

VI. Magíster en Investigación en Administración y Economía de la Empresa, Máster Business Administración con Mención en Marketing, Licenciado en Publicidad, Doctorando Universidad de Valladolid de España, Docente Universidad Eloy Alfaro de Manabí, Manta,Ecuador.
} 


\title{
Resumen
}

El marketing a lo largo de los años ha ido evolucionando para adaptarse a las nuevas exigencias del siglo XXI, de la necesidad de hacer frente a un nuevo tipo de consumidor se da paso a la creación de experiencias de compra que permitan establecer una conexión emocional con los clientes, debido a que el cerebro tiende a tener recuerdos más estables y duraderos cuando estos son propiciados desde las emociones. En este sentido surge el marketing sensorial como una tendencia del marketing destinada a la aplicación de estímulos orientados a los cinco sentidos, los cuales pueden ser implementados tanto en el punto de venta como a través del branding de la marca. Por su parte, el comportamiento de compra hace referencia a diversas características que determinan el accionar del consumidor. La presente investigación utilizó una metodología cuali-cuantitativa con un diseño descriptivo correlacional, mediante la herramienta de encuesta estructurada. Mediante el procesamiento de datos en el programa estadístico SPSS, se determinó de acuerdo a los coeficientes de Kendall, Pearson y Spearman una correlación positiva alta entre las variables de estudio, por lo cual se concluyó que el marketing sensorial influye en el comportamiento de compra de los consumidores de la ciudad de Manta.

Palabras clave: marketing sensorial; sentidos; consumidor; compra; comportamiento.

\begin{abstract}
Marketing over the years has evolved to adapt to the new demands of the XXI century, the need to deal with a new type of consumer gives way to the creation of shopping experiences that allow establishing an emotional connection with the clients, because the brain tends to have more stable and lasting memories when these are propitiated from the emotions. In this sense, sensory marketing emerges as a marketing trend aimed at the application of stimuli oriented to the five senses, which can be implemented both at the point of sale and through branding of the brand. For its part, the purchase behavior refers to various characteristics that determine the actions of the consumer. The present investigation used a qualitative-quantitative methodology with a descriptive correlational design, through the structured survey tool. Through the data processing in the SPSS statistical program, a high positive correlation was determined according to the Kendall, Pearson and Spearman coefficients between the study variables, so it was concluded that sensory marketing influences the purchasing behavior of Consumers in the city of Manta.
\end{abstract}

Key words: sensory marketing; senses; consumer; purchase; behavior. 


\section{Resumo}

$\mathrm{O}$ marketing ao longo dos anos evoluiu para se adaptar às novas exigências do século XXI, a necessidade de lidar com um novo tipo de consumidor dá lugar à criação de experiências de compra que permitem estabelecer uma conexão emocional com os clientes, porque o cérebro tende a ter memórias mais estáveis e duradouras quando estas são propiciadas pelas emoções. Nesse sentido, o marketing sensorial surge como uma tendência mercadológica voltada para a aplicação de estímulos voltados aos cinco sentidos, que podem ser implementados tanto no ponto de venda quanto no branding da marca. Por sua vez, o comportamento de compra refere-se a várias características que determinam as ações do consumidor. A presente investigação utilizou uma metodologia qualitativaquantitativa com delineamento descritivo correlacional, através da ferramenta de pesquisa estruturada. Através do processamento de dados no programa estatístico SPSS, uma correlação positiva alta foi determinada de acordo com os coeficientes de Kendall, Pearson e Spearman entre as variáveis do estudo, assim, concluiu-se que o marketing sensorial influencia o comportamento de compra de Consumidores na cidade de Manta.

Palavras chave: marketing sensorial; sentidos; consumidor compra; comportamento.

\section{Introducción}

El marketing surgió como una respuesta a la necesidad de crear relaciones estables, duraderas y redituables con los clientes, pues la gestión comercial en sus inicios se centraba en la producción, razón por la cual los consumidores se veían limitados a adquirir todo lo que las empresas decidían producir, esto debido a la escasa o nula competencia existente en el mercado, posteriormente, el enfoque se centró en las ventas, en respuesta al leve aumento del número de competidores, todas las empresas buscaban vender y ponían sus esfuerzos en ese objetivo, sin embargo, a raíz del entendimiento de la importancia del cliente, el enfoque comercial fijó su objetivo en este, dando paso al marketing como disciplina.

El marketing se refiere a la creación de relaciones redituables y duraderas en el tiempo con los clientes, en un entorno de alta competitividad, en este sentido se establece el uso de estrategias en torno a las 4 P`s conocidas como las variables del marketing, las cuales establecen una mezcla que orienta las actividades a gestionar para establecer la relación con el cliente. La variable producto es aquella en la que se gestionan todos los aspectos inherentes a la creación, diseño y producción de la oferta al cliente, 
la cual se complementa con la variable precio, siendo esta la variable que gestiona el aspecto redituable de las acciones del marketing, pues esta es la única que genera ingresos, siendo de mucha importancia la gestión de la misma pues a través de esta se solventa la gestión de las demás variables, además de que el precio constituye uno de los principales indicadores de competitividad. Por su parte la variable plaza hace mención a la gestión de la forma en que el producto llega hasta el consumidor, tomando en consideración, la logística, almacenamiento, canales de distribución y puntos de venta. Por último, la variable promoción, la cual determina la forma en que se pone en conocimiento la existencia de la oferta y en donde se deben gestionar aspectos que permitan inducir al cliente a la elección de un producto o marca, la publicidad es uno de los aspectos más relevantes a tener en cuenta dentro de esta variable. (García, Ruiz, \& Escriva, 2014).

Sin embargo, las concepciones básicas del marketing, a raíz del avance tecnológico y el auge de la globalización han debido evolucionar con la finalidad de adaptarse a las nuevas exigencias de los mercados. En este sentido, Gómez \& García (2012) mencionan que las características actuales de los mercados han dado paso a nuevas modalidades en la comercialización, tales como el comercio electrónico, el cual está dejando de lado el antiguo modelo brick \& mortar (venta tradicional) para dar paso a un nuevo modelo, brick $\&$ clic, el cual hace referencia al hecho de que un consumidor no necesita desplazarse hasta un local comercial para adquirir un producto, pues a través de plataformas web, tiene todo al alcance de un clic. Esta realidad ha determinado que las empresas se enfrenten a nuevos retos en el contacto con los clientes, teniendo que idear estrategias que se ajusten a la nueva realidad de la comercialización.

En este nuevo entorno en donde la competencia aumenta de forma acelerada y en donde los consumidores han evolucionado hasta convertirse en consumidores informados, dando paso a un nuevo tipo de consumidor que está cada vez más consciente de las acciones del marketing tradicional, lo cual le condiciona a ser inmune a las estrategias, surge la necesidad de crear nuevas formas de captar y fidelizar a los clientes, en este contexto surgen las nuevas tendencias del marketing, las cuales direccionan nuevos aspectos que complementan al marketing tradicional.

Dentro de estas nuevas tendencias se encuentra el neuromarketing, el cual se centra en la utilización de las neurociencias para el estudio y comprensión del comportamiento del consumidor. De acuerdo a Coca (2008), el neuromarketing corresponde a una disciplina orientada al estudio del consumidor a 
través de las neurociencias, las cuales se encargan del estudio de la estructura y la función química, farmacología, y patología del sistema nervioso y de cómo sus diferentes elementos interaccionan y dan origen a la conducta, esto conlleva a una comprensión de la misma a través del análisis de los procesos químicos y neuronales.

De acuerdo a Baptista et al. (2010), la utilización del neuromarketing plantea un nuevo enfoque en el estudio del consumidor, pues los resultados obtenidos tendrán base científica neurológica, considerando que las reacciones neuronales y químicas determinará la eficiencia de los estímulos de marketing de forma objetiva, contrario a otros métodos de estudio del consumidor, en donde los resultados están determinados por la interpretación subjetiva de los investigadores, lo cual ha acarreado que actividades de marketing resulten poco efectivas en la captación de clientes.

Esta nueva tendencia constituye un amplio potencial debido al enfoque de entendimiento a través de la evaluación de las reacciones a los estímulos de marketing para comprender lo que resulta importante para el consumidor, no desde lo que este dice, sino desde sus reacciones cerebrales, lo cual supone una eficiencia superior en el estudio del comportamiento.

Así, se determina la importancia del neuromarketing, como un nexo entre la necesidad de comprender la eficiencia de las actividades de marketing tradicional y el nuevo enfoque de crear estrategias en base a ese conocimiento obtenido. Dentro de este contexto, la información obtenida a través del neuromarketing, conllevó a la comprensión de que el nuevo consumidor requiere de nuevos métodos de seducción, ampliando su necesidad a la obtención de experiencias en los procesos de compra.

Esta comprensión ha dado paso al marketing de experiencia, el cual implica el evocar reacciones emocionales para crear mejores relaciones con el cliente, surge como una necesidad creciente de crear momentos y emociones únicas como medio para hacer frente a la competencia y alcanzar mayor captación de clientes. (Molinillo et al. 2015).

En este sentido, esta tendencia considera la creación de experiencias de consumo en donde el consumidor converge en sus aspectos cognitivos, emocionales y afectivos en relación a la marca o producto, determinando la elección, compra y fidelización para con el mismo. Al respecto, Jiménez \& Campo (2016) indican que el marketing de experiencia se basa en la creación de una comunicación 
recíproca con el consumidor bajo el entendimiento de que las decisiones que esta toma no se limita al raciocinio, sino que las emociones pueden jugar un papel de mucha importancia.

La importancia del análisis del comportamiento del consumidor sale a relucir en torno a cualquier tendencia del marketing, en este contexto la psicología del consumidor se convierte en una disciplina altamente necesaria como medio de contextualización de las características del consumidor, pues resulta imperativo conocer las motivaciones que lo orientan hacia una compra, con el fin de potenciar dicha compra en una relación de fidelidad. (Echeverry \& Sandoval, 2011).

En consideración, Segura \& Sabaté (2008) destacan la importancia del marketing de experiencia bajo la premisa de que el nuevo consumidor fija sus percepciones más allá de la simple relación costobeneficio en la adquisición de un producto, sino que su elección estará determinada por las emociones que despierte en sí el proceso de compra, las emociones y las vivencias a las que le lleve el consumo.

Bajo este contexto, se determina la importancia de los sentidos en el proceso de despertar emociones y crear experiencias de consumo lo cual permitirá inducir no solo una compra inmediata sino también las relaciones a largo plazo. Este entendimiento da paso al surgimiento del marketing sensorial, como una actividad que constituye el centro de la presente investigación.

El marketing sensorial hace referencia a la aplicación de estímulos a los sentidos (vista, oído, olfato, gusto y tacto), tanto en el punto de venta como a través del branding, con la finalidad de orientar el comportamiento del consumidor desde la estimulación emocional y de experiencias.

Figura 1: Proceso de marketing sensorial

\begin{tabular}{|c|c|c|c|}
\hline Factores sensoriales & Percepciones & \multicolumn{2}{|c|}{ Reacciones } \\
\hline $\begin{array}{l}\text { Relativos al punto de venta } \\
\text { Vista } \\
\text { Tacto } \\
\text { Olfato } \\
\text { Oído } \\
\text { Gusto }\end{array}$ & Cognitivas & De actitud & $\begin{array}{l}\text { Imagen de marca } \\
\text { Satisfacción } \\
\text { Fidelidad }\end{array}$ \\
\hline $\begin{array}{l}\text { Relativas al producto } \\
\text { Vista } \\
\text { Tacto } \\
\text { Olfato } \\
\text { Oído } \\
\text { Gusto }\end{array}$ & Emocionales & De comportamiento & $\begin{array}{l}\text { Frecuencia } \\
\text { Tiempo } \\
\text { Circuitos } \\
\text { Categorías } \\
\text { Nivel de gusto }\end{array}$ \\
\hline
\end{tabular}

Fuente: (Manzano, et. al, 2012, p.77) 
Este proceso del marketing sensorial muestra las diferentes consideraciones con respecto de la aplicación de estímulos sensoriales, así, los estímulos en el punto de venta desarrollan percepciones cognitivas las cuales conllevan a una reacción de actitud, mientras que los estímulos sensoriales relativos al producto generan percepciones emocionales que determinan una reacción de comportamiento. En este proceso se puede ver plasmada la importancia de la aplicación de estrategias de marketing sensorial.

Álvarez del Blanco (2011) afirma que los sentidos en relación a la exposición de las marcas reaccionan en estas medidas: el sentido visual reacciona 58\%, el olfativo 45\%, el auditivo $41 \%$, el gusto $31 \%$ y el táctil 25\%. Los sentidos son la fuente que recolecta la información del entorno y lleva al cerebro para su interpretación, esto contempla que las valoraciones ante presentadas planteen un alto potencial en la gestión de los sentidos con el fin de captar y fidelizar a los clientes.

El presente estudio se enfoca en los consumidores de la ciudad de Manta, esto debido al amplio sector comercial que se desarrolla en diversos puntos de la ciudad como resultado del terremoto ocurrido en el año 2016, el cual afectó al casco comercial y determinó que las zonas de comercio se diversificaran dando paso a nuevas y crecientes plazas comerciales, aumentando así la competitividad interna. Según un informe presentado por el INEC, en Manta existen alrededor de 9 mil establecimientos comerciales, los cuales representan 27,8\% del total de establecimientos en toda la provincia de Manabí, lo que convierte a la ciudad en un territorio con amplio potencial económico tanto para el estudio como para la aplicación de estrategias de marketing.

La presente investigación tiene como finalidad determinar si existe una correlación entre las variables de estudio, dando paso a la concepción de la influencia de los sentidos en el comportamiento de compra de los consumidores de la ciudad de Manta.

\section{Marco conceptual de la investigación}

La realidad del siglo XXI, con sus cambios acelerados y el auge tecnológico han determinado que las actividades comerciales gestionadas a través de los enfoques del marketing, planteen nuevos retos para las empresas que buscan destacar ante la competencia. En este sentido el marketing sensorial evidencia un alto potencial para la captación y fidelización de clientes. 
Barrios (2012) plantea como concepto del marketing sensorial como aquella actividad que se involucran los sentidos con la finalidad de influir el comportamiento de los consumidores, permitiendo evaluar la calidad percibidad y las percepciones que generan.

Por su parte, De Garcillán López-Rúa (2015), plantea que el objetivo del marketing sensorial es evaluar las reacciones que genera un producto en las personas, tomando como medio a los sentidos, utilizando uno o varios a la vez.

En el enfoque filosófico, Lindstrom (2010), a través de sus diversos estudios planteó una filosofía enfocada en que, para lograr la máxima eficacia en la aplicación de marketing sensorial, se debe utilizar una mezcla sensorial involucrando varios de los sentidos, bajo la premisa de que mientras haya más asociación de sentidos, más se verá influenciada la conducta del consumidor.

En este sentido, el comportamiento de compra del consumidor se refiere a la conducta en el proceso que sigue el consumidor desde el reconocimiento de la necesidad, hasta la compra y ante la posibilidad de una posible recompra, este proceso puede verse influenciado por diversas características y circunstancias tanto externas (estímulos) o internas (emociones, percepciones). (Coca, 2010).

\section{Metodología}

Se utilizó una metodología de tipo cuali-cuantitativa debido a la recolección de datos numéricos en relación a las variables descritas de forma cualitativa. El diseño se planteó de forma descriptiva y correlacional, de acuerdo a Cruz et al. (2014), la investigación descriptiva se implementa cuando se estudia y describe el comportamiento de un sujeto sin influir en el mismo, por su parte el enfoque correlacional hace referencia a que se compruebe o no la relación existente entre las variables de estudio.

\section{Población}

Para propósitos del estudio, se tomará en consideración a un total de 113,465 como miembros de la población económicamente activa, los cuales corresponden al 50.1\% de la población total de la ciudad de Manta $(226,477)$ de acuerdo al INEC. 


\section{Muestra}

Se utilizó un muestreo probabilístico simple, debido a la homogeneidad de la población de estudio. Con la aplicación de la fórmula de población finita, determinando un porcentaje de fiabilidad del $95 \%$ y un 5\% de margen de error, se obtuvo una muestra de 384 personas a encuestar en la ciudad de Manta.

\section{Instrumentos}

Para la recolección de los datos se utilizó un instrumento desarrollado por los investigadores en donde se evalúan las siguientes dimensiones e indicadores:

Tabla 1. Operacionalización de variables

\begin{tabular}{|c|c|c|}
\hline Variable & Dimensión & Indicador \\
\hline \multirow{7}{*}{ Marketing sensorial } & \multirow{5}{*}{ Los sentidos } & Sentido visual \\
\hline & & Sentido auditivo \\
\hline & & Sentido olfativo \\
\hline & & Sentido del gusto \\
\hline & & Sentido del tacto \\
\hline & \multirow{2}{*}{ Emociones } & Asociación \\
\hline & & Reacción \\
\hline \multirow{4}{*}{$\begin{array}{l}\text { Comportamiento de } \\
\text { compra }\end{array}$} & Pre compra & Motivación de compra \\
\hline & & Evaluación de alternativas \\
\hline & Compra & Decisión de compra \\
\hline & Post Compra & $\begin{array}{l}\text { Experiencia de consumo } \\
\text { Recompra }\end{array}$ \\
\hline
\end{tabular}

Fuente: Autores

Este instrumento está compuesto por 33 ítems con tipo de respuesta escala Likert en un rango de 1 a 5 , siendo 1 totalmente en desacuerdo, 2 en desacuerdo, 3 ni de acuerdo ni en desacuerdo, 4 de acuerdo y 5 totalmente de acuerdo.

\section{Resultados}

\section{Análisis de fiabilidad}

Los datos recabados mediante la herramienta de encuesta estructurada, se procesaron en el programa estadístico SPSS. Con la finalidad de evidenciar la fiabilidad del instrumento de investigación utilizado a continuación se presenta la valoración del coeficiente de Cronbach obtenido: 
Tabla 5. Análisis de fiabilidad

\begin{tabular}{cc}
\hline \multicolumn{3}{c}{ Estadísticas de fiabilidad } \\
\hline Alfa de Cronbach & N de elementos \\
0,904 & 33 \\
\hline
\end{tabular}

Fuente: Análisis Software IBM SPSS

El coeficiente Alfa de Cronbach determina el grado de fiabilidad del instrumento mediante la evaluación de la pertinencia de los datos recabados. Esta valoración puede variar de 0 a 1 y el grado de confiabilidad se da bajo la premisa de que, mientras más se acerque a 1, mayor es el grado de confiabilidad. En el caso de la presente investigación, se obtuvo un indicador de 0,90, lo que es superior al mínimo requerido, por tanto, se sustenta la aplicabilidad del instrumento.

\section{Análisis correlacional}

Se efectuó un análisis correlacional bivariado, mediante los coeficientes de Kendall, Pearson y Spearman. Estos coeficientes pueden arrojar valoraciones de 0 a 1 , el grado de correlacionalidad está determinado bajo el contexto de que la correlación será más significativa en tanto más se acerque al valor máximo de 1. Las categorías inherentes a los rangos de relación son los siguientes:

Tabla 3. Rangos de correlación

\begin{tabular}{cc}
\hline Rango correlación & Valoración \\
\hline$(0.00,0.20)$ & Muy baja Correlación \\
{$[0.20,0.40)$} & Baja correlación \\
{$[0.40,0.60)$} & Moderada correlación \\
{$[0.60,0.80)$} & Alta correlación \\
{$[0.80,1.00)$} & Muy alta correlación \\
\hline
\end{tabular}

Fuente: Software IBM SPSS

\section{Coeficiente de Kendall}

De acuerdo a los datos obtenidos correspondiente al coeficiente de Kendall, este presenta una correlación de 0,80 entre las variables marketing sensorial y comportamiento de compra, lo que implica que, de acuerdo con los rangos valorativos mostrados anteriormente, existe una correlación 
positiva alta entre las variables estudiadas, lo que responde al objetivo de la investigación, evidenciando la influencia del marketing sensorial en el comportamiento de compra.

Tabla 4. Coeficiente correlación de Kendall

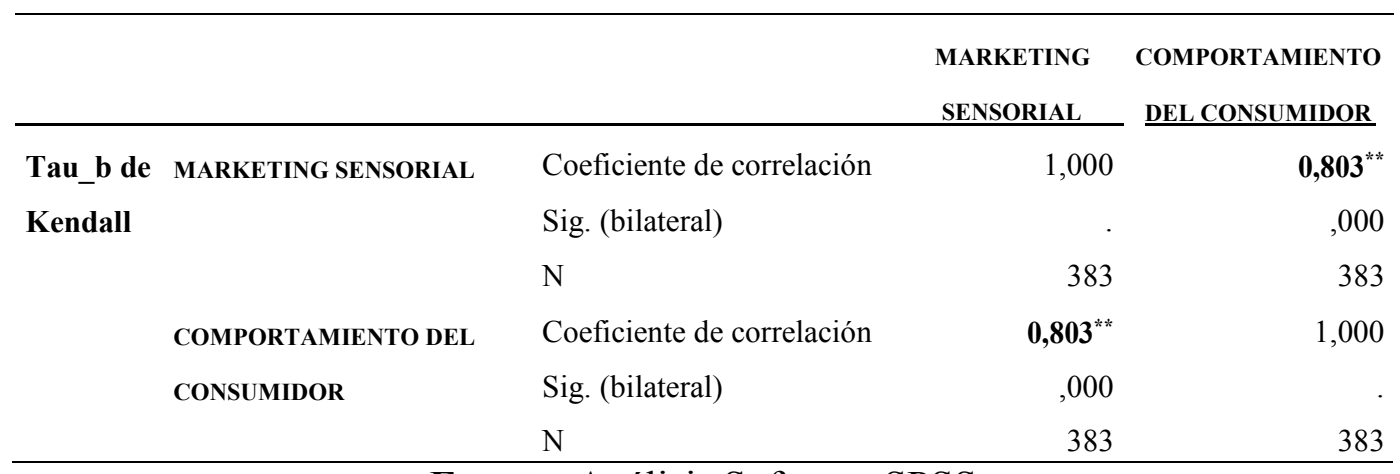

Fuente: Análisis Software SPSS

\section{Coeficiente de Pearson}

Por su parte, de acuerdo al coeficiente de Pearson, el valor de correlación entre el marketing sensorial y el comportamiento de compra es de 0,798 , lo que se puede ajustar a 0,80. Este valor muestra una variación mínima en relación al coeficiente antes analizado, por lo que se sustenta la correlación positiva alta entre las variables estudiadas.

Tabla 5. Coeficiente correlación de Pearson

\begin{tabular}{llrr}
\hline & & $\begin{array}{c}\text { MARKETING } \\
\text { SENSORIAL }\end{array}$ & $\begin{array}{c}\text { COMPORTAMIENTO } \\
\text { DEL CONSUMIDOR }\end{array}$ \\
\hline MARKETING SENSORIAL & Correlación de Pearson & 1 & $\mathbf{0 , 7 9 8}^{* *}$ \\
& Sig. (bilateral) & 380 \\
COMPORTAMIENTO DEL & $\mathrm{N}$ & 383 & 383 \\
CONSUMIDOR & Correlación de Pearson & $\mathbf{0 , 7 9 8}^{* * *}$ & 1 \\
& Sig. (bilateral) &, 000 & 383 \\
\hline
\end{tabular}

Fuente: Análisis Software SPSS

\section{Coeficiente de Spearman}

En relación al coeficiente de Spearman, el valor obtenido de correlación entre las variables analizadas es de 0,804 , evidenciando una valoración correspondiente con los otros dos coeficientes ya planteados. 
Esto indica que la correlación positiva alta entre el marketing sensorial y el comportamiento de compra es significativa.

Finalmente, de acuerdo al análisis de correlación se determina que, los tres coeficientes analizados mediante el programa estadístico SPSS en la modalidad de correlación bivariada, presentan valores que no difieren en gran magnitud, lo que determina que la relación entre las variables es significativamente constante.

\section{Prueba de hipótesis}

Se aplicó el coeficiente estadístico Chi Cuadrado (x2), el cual se sustenta en el supuesto de que las variables no están relacionadas, dando paso a la hipótesis nula (H0), si el resultado obtenido es mayor a 0,05, esta hipótesis nula se acepta, $x^{\wedge} 2>0,05$ (independencia de variables), mientras que, si el resultado es menor a 0,05 , se aprueba la hipótesis alternativa $(\mathrm{H} 1), \mathrm{x}^{\wedge} 2<0,05$ (variables relacionadas).

En este estudio, se plantean las siguientes hipótesis:

H0: El marketing sensorial no influye en el comportamiento de compra de los consumidores de la ciudad de Manta.

H1: El marketing sensorial influye en el comportamiento de compra de los consumidores de la ciudad de Manta.

Tabla 6. Prueba de hipótesis general - Chi cuadrado

\begin{tabular}{lcrrr}
\hline \multicolumn{4}{c}{ Pruebas de chi-cuadrado } \\
\hline $\begin{array}{c}\text { Marketing sensorial / Comportamiento de } \\
\text { compra }\end{array}$ & Valor & df & \multicolumn{2}{c}{$\begin{array}{c}\text { Significación asintótica } \\
\text { (bilateral) }\end{array}$} \\
Chi-cuadrado de Pearson & 1957,177 & 459 & $\mathbf{0 , 0 0 0}$ \\
Razón de verosimilitud & 975,844 & 459 &, 000 \\
Asociación lineal por lineal & 87,048 & 1 &, 000 \\
N de casos válidos & 383 & & \\
\hline
\end{tabular}

Fuente: Análisis Software IBM SPSS

La significancia obtenida de 0,00 , de acuerdo a las indicaciones del coeficiente, conllevan a que se descarte la hipótesis nula, aceptando la hipótesis alternativa: El marketing sensorial influye en el comportamiento de compra de los consumidores de la ciudad de Manta, lo que le da mayor 
sustentabilidad a los grados de correlación de variables encontrados en los coeficientes presentados en el apartado anterior.

\section{Discusión}

La presente investigación plantea un referente para el estudio de las características del comportamiento del consumidor mantense, debido a que existen escasos estudios formales orientados a la comprensión de su accionar y la relevancia que tienen las actividades de marketing en los mismos.

Desde el punto de vista investigativo, los resultados encontrados plantean una pauta para el desarrollo de nuevas investigaciones que amplíen las dimensiones evaluadas para evidenciar nuevos resultados en torno a diferentes temáticas, pues se busca que este estudio forje un interés para la continuidad de nuevas líneas de investigación enfocadas en las nuevas tendencias del marketing y las características que determinan el comportamiento de compra del consumidor.

En relación a la practicidad del estudio planteado, está orientado a brindar un aporte en el direccionamiento de estrategias para el sector comercial de la ciudad de Manta, a través del descubrimiento de la importancia que brindan los consumidores mantenses a los estímulos sensoriales y las creaciones de experiencias de consumo a través de las emociones.

\section{Conclusiones}

El marketing sensorial nace como una respuesta al surgimiento del nuevo consumidor que se caracteriza por ser un consumidor informado que está cada vez más consciente de las actividades de marketing tradicional, desarrollando inmunidad a sus estrategias. La necesidad de crear experiencias en los consumidores que se estimules desde el sentido emocional de las personas a través del uso de estímulos sensoriales, determina que esta nueva tendencia esté en auge.

En la investigación se concluye que existe una correlación positiva alta, la cual es significativa y constante pues se sustentó a través de tres coeficientes de correlación, mediante el análisis estadístico en el programa SPSS, además de la prueba de hipótesis mediante el coeficiente de Chi-cuadrado. Estas valoraciones determinan que, en la ciudad de Manta, Ecuador, el marketing sensorial sí influye en el comportamiento de compra de los consumidores. 


\section{Referencias Bibliográficas}

Álvarez del Blanco, R. (2011). Branding hoy: estrategias que funcionan. Marca multisensorial, espléndidamente. Marketing \& Ventas, 106 (1), 26 - 33.

Baptista, M., Del Fátima, M., \& Mora, C. (2010). Neuromarketing: Conocer al cliente por sus percepciones. TEC Empresarial, 4 (3), 9-19.

Barrios, M. (2012). Marketing de la Experiencia: principales conceptos y características. Palermo Business Review, 7 (1), 67-89.

Coca, A. (2008). El concepto de Marketing: pasado y presente. Revista de Ciencias Sociales, 14 (2), 391-414.

Coca, A. (2010). Neuromarketing: Las emociones y el comportamiento de compra. Perspectivas, (25), 9-24.

Cruz, C., Olivares, S., \& González, M. (2014). Metodología de la Investigación. México: Grupo Editorial Patria.

De Garcillán López-Rúa, M. (2015). Persuasión a través del marketing sensorial y experiencial. Opción, 31 (2), 463-478.

Echeverry, I., \& Sandoval, M. (2011). Análisis del comportamiento de compra en un contexto experimental simulado. Suma Psicológica, 18 (2), 49-63.

Garcia, A., Ruiz, C., \& Escriva, J. (2014). Marketing en la actividad comercial. Madrid: Mc Graw Hill Education.

Gómez, M., \& García, C. (2012). Marketing sensorial. Distribución y consumo, 122 (1), 30-40.

INEC. (abril de 2018). Instituto Nacional de Estadísticas y Censos. Ecuador en cifras.

Jiménez, J., \& Campo, S. (2016). Marketing Experiencial en FITUR: Análisis de dos destinos competidores, Islas Canarias e Islas Baleares. Pasos, 14 (1), 75-91.

Lindstrom, M. (2010). Ventas para los sentidos. En M. Lindstrom, Buyology: verdad y mentiras sobre por qué compramos (págs. 147-169). Barcelona: Booket.

Manzano, R., Gavilán, D., Avello, M., abril, C., \& Serra, T. (2012). Marketing sensorial (comunicar con los sentidos en el punto de venta). Madrid: Pearson Educación.

Segura, C., \& Sabaté, F. (2008). Marketing Experiencial: el marketing de los sentimientos y sus efectos sobre la mejora en la comunicación. XII Congreso de Ingeniería de Organización (págs. 267272). Burgos: II International Conference on Industrial Engineering and Industrial Management. 